\title{
Salidroside Ameliorates Chronic Intermittent Hypoxia-induced Endothelial Insulin Resistance via Suppression of ERK1/2 Activation
}

\author{
Linyi Li, Yunyun Yang, Xinliang Ma, Yongxiang Wei, Yanwen Qin
}

Key Laboratory of Upper Airway Dysfunction-related Cardiovascular Diseases, Beijing Anzhen Hospital, Capital Medical University, Beijing Institute of Heart, Lung and Blood Vessel Diseases, China

Backgroud: Obstructive sleep apnea (OSA), a condition leading to chronic intermittent hypoxia (CIH), is an independent risk factor for cardiovascular disease and type 2 diabetes and is correlated with insulin resistance. Insulin stimulates production of nitric oxide (NO) in vascular endothelial cells through the IRS-1/PI3K/Akt/eNOS pathway (IRS-1, insulin receptor substrate 1; PI3K, phosphatidylinositol 3-kinase; eNOS, endothelial NO synthase). We wondered if $\mathrm{CIH}$ that mimic OSA affects insulin signalling/action both in the aortas of C57BL/6J mice and in the human umbilical vein endothelial cells (HUVECs), and whether salidroside (Sali), a major active constituent of Rhodiola sachalinensis, could reverse this inhibitory effect.

Methods: Forty male C57BL/6J mice were randomly divided into four groups ( $\mathrm{n}=10 /$ group): (1) control (intermittent air), (2) $\mathrm{CIH}$ (21\%-5\%, $90 \mathrm{~s} /$ cycle, $10 \mathrm{~h} /$ day), (3) CIH+50 mg/kg Sali, (4) CIH+100 mg/kg Sali, 7 weeks intervention. HUVECs were treated with $10 \mathrm{uM}$ or $100 \mathrm{uM}$ of Sali and exposed to CIH (21\%-5\%, $1 \mathrm{~h} /$ cycle) for $24 \mathrm{~h}$ or $48 \mathrm{~h}$ with or without insulin. Blood pressure, endothelium-dependent relaxation (EDR) and insulin-stimulated activation of signal molecules were assessed.

Results: In vivo, we observed that $\mathrm{CIH}$ increased systolic blood pressure and impaired EDR of aortas in C57BL/6J mice. $\mathrm{CIH}$ increased IRS-1 phosphorylation at Ser307 and Ser612, impaired insulin-stimulated phosphorylation of IRS-1 at Tyr896 and Akt/eNOS pathway in aortas. In addition, CIH increased extracellular signal-regulated kinase 1/2 (ERK1/2) phosphorylation. Treatment of C57BL/6J mice with Sali dose-dependently ameliorated theses deleterious effects of CIH. In vitro, CIH impaired IRS-1/PI3K/Akt/eNOS pathway that resulted in reduced insulin-stimulated NO production in HUVECs. These events were accompanied by elevated ERK1/2 phosphorylation and were reversed by ERK1/2 inhibition. Sali improved endothelial insulin resistance by inhibition of ERK1/2 activation, leading to positive regulation of serine/tyrosine phosphorylation of IRS-1 and restoration of downstream Akt/eNOS activation and insulin-mediated NO production.

Conclusion: Our data suggest that CIH may cause endothelial insulin resistance by dysregulation of IRS-1 serine/tyrosine phosphorylation via ERK1/2. Salidroside is effective in ameliorating $\mathrm{CIH}$-induced insulin resistant endothelial dysfunction by inhibition ERK1/2 activation and beneficial regulation of IRS-1 function. 